\title{
Belgeo
}

Revue belge de géographie

$1 \mid 2018$

Miscellaneous

\section{Stephen Smith, La Ruée vers l'Europe. La jeune Afrique en route pour le Vieux Continent}

Samuel Lietaer

\section{(2) OpenEdition}

1 Journals

Édition électronique

URL : http://journals.openedition.org/belgeo/29709

DOI : 10.4000/belgeo.29709

ISSN : 2294-9135

Éditeur :

National Committee of Geography of Belgium, Société Royale Belge de Géographie

Référence électronique

Samuel Lietaer, «Stephen Smith, La Ruée vers l'Europe. La jeune Afrique en route pour le Vieux Continent », Belgeo [En ligne], 1 | 2018, mis en ligne le 18 décembre 2018, consulté le 23 septembre 2020. URL http://journals.openedition.org/belgeo/29709; DOI : https://doi.org/10.4000/belgeo.29709

Ce document a été généré automatiquement le 23 septembre 2020

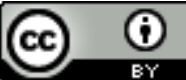

Belgeo est mis à disposition selon les termes de la licence Creative Commons Attribution 4.0 International. 


\title{
Stephen Smith, La Ruée vers l'Europe. La jeune Afrique en route pour le Vieux Continent
}

\author{
Samuel Lietaer
}

\section{RÉFÉRENCE}

Stephen Smith, La Ruée vers l'Europe. La jeune Afrique en route pour le Vieux Continent, Grasset, Paris, 2018, 272 p.

\section{Introduction}

1 Ancien reporter spécialiste de l'Afrique (1986-2005) pour Reuters, RFI, Libération, et Le Monde, Stephen Smith s'intéresse depuis une trentaine d'années aux migrations de l'Afrique vers l'Europe.

2 Smith influence incontestablement le débat public avec son dernier ouvrage, paru en février 2018, au titre évocateur: La ruée vers l'Europe. La jeune Afrique en route pour le Vieux Continent. Sa principale thèse avance que le développement économique de l'Afrique alimente les migrations massives vers l'Europe. La vaste couverture médiatique dont a joui cet essai après sa parution montre la pertinence du sujet pour une Europe en « crise migratoire ».

3 Auteur de 13 livres en français, Smith connaît la formule des ouvrages grand public, médiatisés et couronnés de succès ${ }^{1}$. Ce dernier ouvrage a été cité par le président français lors du débat célébrant sa première année au pouvoir. Il a notamment reçu le prix du Livre Géopolitique 2018, remis par le Ministre des Affaires Étrangères. Les critiques d'académiques viennent seulement après avoir atteint le "grand public ", éventuellement son opinion, et les décideurs politiques. Si ces contre-argumentaires 
ont tardé à paraître, ils sont finalement abondants (e.a. Héran, 2018a, 2018b ; Bréville, 2018 ; Brachet \& Scheele, 2018).

4 Après avoir résumé la thèse de l'essai et son apport à la littérature, cette recension soulève les principales critiques «méthodologiques» dans l'objectif de le situer dans son contexte académique et politique. Ce compte rendu critique épingle certains éléments «factuels » et discursifs de l'ouvrage qui le caractérisent en les confrontant à la littérature académique.

\section{La thèse de l'essai : la jeune Afrique va submerger le Vieux continent}

\section{L'importance démographique et économique}

5 L'essai s'organise en cinq chapitres au style très accessible, malgré les nombreux chiffres articulés autour de la démographie et - en moindre mesure - du développement économique. Si le titre alarmiste et fataliste fait référence à la « crise migratoire » en Europe, les sujets touchés sont en effet bien plus nombreux et bien plus vastes. La démographie, avec un taux de croissance élevé, vient se télescoper avec une pauvreté persistante, les conflits armés, la montée des extrémismes religieux, les défis sanitaires, urbains, économiques, l'affrontement entre les générations. Mais la plupart de ces sujets ne sont que très partiellement abordés.

6 Avec une approche "sans tabous ", Smith prétend traiter ce thème principalement du point de vue africain, à partir d'un nombre de réalités africaines choisies. Smith prend le contre-pied du discours dominant sur " l'émergence africaine " ${ }^{2}$. Il navigue plutôt de l'afro-optimisme naïf des années 2000 vers l'afro-pessimisme désespérant des années 1990 : "L'Afrique est le seul continent ayant perdu sur les deux tableaux de la mondialisation: ses disparités internes se sont accrues encore plus qu'ailleurs sans que le continent ait gagné du terrain par rapport au niveau de vie dans les pays développés (...)» (pp. 140-141).

7 Sa thèse principale est contre-intuitive: la jeune Afrique va submerger le Vieux Continent parce qu'elle serait soumise à un "rouleau compresseur démographique " alimenté par le développement économique et la fécondité très élevée au sud du Sahara. Cette exceptionnelle jeunesse africaine, déjà mondialisée mais souvent sans accès à cette mondialisation en fait la matrice de l'avenir. La part des moins de 15 ans représente actuellement plus de $40 \%$ de la population africaine - contre $16 \%$ en Europe (p. 20)3.

D'après des estimations des Nations unies que Smith a utilisées, sa population passera de 1,2 milliard d'habitants en 2017 à 2,5 milliards en 2050, et même à 4,4 milliards en 2100. Pendant ce temps, le continent connaîtra un important développement économique, les revenus des habitants augmenteront, et un nombre croissant d'entre eux disposeront « des moyens nécessaires pour aller chercher fortune ailleurs » (p. 18).

9 En couverture du livre et dans l'introduction, l'auteur avance que la migration subsaharienne se déversera sur l'Europe d'ici trente ans au risque de ruiner notre protection sociale. Dans les pages finales, Smith formule sa thèse de manière un peu plus nuancée, sans que ce « déversement » paraisse certain. 


\section{Deux conditions majeures pour déclencher « la ruée »}

10 Smith soutient que pour déclencher la « ruée vers l'Europe » il faut que « deux conditions majeures » soient réunies, à savoir que les réels candidats au départ aient suffisamment de ressources économiques d'une part et puissent s'appuyer sur des réseaux sociaux d'autre part. Partant de cette "loi des grands nombres» et de la croissance démographique, il faudrait donc s'attendre à une « levée en masse » du continent, à tel point que, dans trente ans, « 20 à $25 \%$ de la population européenne sera d'origine africaine », contre 1,5 à $2 \%$ en 2015, soit 9 millions de personnes (pp. 18, 45).

11 La première condition est donc «le franchissement d'un seuil de prospérité minimale par une masse critique d'Africains sur fond de persistance d'une grande inégalité de revenus entre l'Afrique et l'Europe. La force d'attraction de l'extérieur s'exercera alors pleinement sur une multitude de jeunes sans perspectives d'emploi, mais capables de réunir, avec l'aide de leurs parents au sens large, le pactole de départ nécessaire pour relever les défis d'un voyage souvent clandestin» (p. 143).

12 La seconde condition majeure pour qu'un "saut quantique" se produise dans les migrations vers l'Europe est "l'existence de communautés diasporiques, qui constituent autant de têtes de pont sur l'autre rive de la Méditerranée » (p. 144).

13 À ces deux conditions, il ajoute "une circonstance aggravante", à savoir le "stress écologique » qui s'y ajoutera pour amplifier le mouvement dans certaines parties du continent (p. 143). Ce dernier déterminant n'est pas développé davantage.

14 L'auteur tire des conclusions politiques de deux paradoxes qu'il avance dans son livre:

15 1. «La difficulté d'une diaspora à se "fondre» au sein d'une population d'accueil prolonge son efficacité comme « cellule d'accueil » pour les nouveaux immigrants (...) » (p. 145).

2. «Les pays du Nord subventionnent les pays du Sud, moyennant l'aide au développement, afin que les démunis puissent mieux vivre et - ce n'est pas toujours dit aussi franchement - rester chez eux. Or, ce faisant, les pays riches se tirent une balle dans le pied. (...) C'est l'aporie du « codéveloppement ", qui vise à retenir les pauvres chez eux alors qu'il finance leur déracinement » (pp. 147-148).

\section{Le problème du « vivre-ensemble »}

L'ancien journaliste évoque les actuels et potentiels problèmes de "vivre-ensemble » des sociétés qui ont fait des choix migratoires très différents, à savoir les États-Unis (culturellement hyper-diversifiés) et le Japon qui continue une politique migratoire fermée, malgré sa pyramide démographique inversée (pp.194-203) : «Le Japon et les États-Unis ne sont ni l'enfer ni le paradis sur terre pour avoir fait des choix inverses sur la diversité dans leur population. Ils marquent deux points éloignés l'un de l'autre dans un continuum où chaque communauté nationale doit trouver son point d'équilibre » (p. 202).

17 Il y mobilise la thèse du livre du politologue Robert Putnam «Bowling Alone » (2000). Chiffres à l'appui, cette étude avance que le capital social au sein de la société américaine était en chute libre depuis les années 1960. La proportionnalité directe entre le «bonding capital » et le "bridging capital » demanderait alors, selon Smith, « un point d'équilibre en matière d'immigration. Car, sans immigration, il n'y a ni défi lancé aux habitudes invétérées ni plaisir de retrouver ses façons habituelles de faire » (p. 200). 

sentent "envahis", ils coupent les ponts, rentrent dans leurs cavernes identitaires et se muent en troglodytes. La victoire électorale de Donald Trump semble indiquer que c'est le cas d'un nombre important d'Américains » (p. 200).

années à venir. L'auteur y dévoile plus clairement sa position idéologique et politique. Ainsi, Smith rappelle que :

• «La migration massive d'Africains vers l'Europe n'est dans l'intérêt ni de la jeune Afrique ni du Vieux Continent » (p. 223);

- Les non-Européens noirs et arabes dérangent inévitablement les Européens blancs (pp. 182, 212);

-A l'avenir, les «bons augures » pour l'Afrique seront «de funestes présages pour l'Europe » (p. 225);

- «L'union forcée entre la jeune Afrique et le Vieux Continent n'est pas encore une fatalité. Il y a de la marge pour des choix politiques» (p. 225);

- «L'Eurafrique signifiera la fin de la sécurité sociale en Europe (...) » (p. 227);

- "L'évident abus du droit d'asile est minimisé en se mettant "à la place des pauvres gens" " (p. 230).

Son message: la «ruée» de l'Afrique vers l'Europe est un phénomène qui peut survenir, mais qui n'est pas encore vraiment enclenché. L'auteur suggère que face à sa prédiction d'une invasion de l'Europe par les "nouveaux barbares ", le seul salut possible passe par les «bons choix politiques». Un exemple qui rappelle clairement le programme de certains partis politiques européens est le suivant: "seule l'entrée très sélective de quelques bras et, surtout, de cerveaux africains apporterait des avantages à l'Europe » (p. 223).

En effet, il remet en question « une gestion "souple" des flux migratoires » qui " engage un pari sur l'avènement d'une vraie prospérité en Afrique, semblable à celle qui fait désormais repartir dans leur pays d'origine des Mexicains des États-Unis. (...) Il suffira de tenir, tant bien que mal, pendant deux ou trois générations. Sera-ce possible en cas de ruée vers l'Europe?» (p. 235). Néanmoins, après un discours en faveur d'une "Europe forteresse », il termine paradoxalement par se demander à quoi ressemblerait « une Afrique qui bénéficierait de toute cette énergie actuellement mobilisée pour lui tourner le dos » (p. 236).

\section{Critiques}

\section{Quel apport à la littérature?}

L'ouvrage discuté - a priori séduisant - ne peut se profiler comme une étude de compréhension des dynamiques des migrations entre l'Afrique et l'Europe. En effet, derrière des chiffres et des rapports mal interprétés qui enfument le lecteur avec une démonstration d'ensemble anxiogène se cache un projet politique. Si l'auteur ne prétend pas vouloir contribuer à combler les lacunes de la «tragédie statistique de l'Afrique »(p. 34) ${ }^{4}$, le problème réside dans le fait que sa démonstration démographique parait implacable, alors qu'elle est basée sur des interprétations parfois fallacieuses des données chiffrées de rapports (cf. Héran, 2018 ; ci-dessous).

En outre, Smith manipule le lecteur non averti par une sélection biaisée de références qui conviennent à son propos, et à l'inverse, n'intègre pas les références académiques 
qui le gênent. Ce n'est donc pas une manière appropriée de combler le manque de réflexion et d'intérêt vis-à-vis de ce sujet complexe dans les milieux politiques et académiques (cf. ci-dessous).

La perspective de géographie humaine recherchée par l'auteur - analyser les migrations de l'Afrique vers l'Europe au prisme de la démographie et du développement - n'est pas neuve. L'approche «utilitaire» de Smith n'entre pas aisément dans les cases théoriques de la littérature. Le lecteur remarque qu'il navigue entre tendance « volontariste » (agency) et « structuraliste » (structure).

Parmi les exemples empiriques de ces modèles, on peut citer les transferts de fonds et les investissements communautaires induits par les migrations, versus la fuite des cerveaux, la dépendance et l'inégalité induites par les migrations. La migration y est généralement présentée respectivement soit comme une solution, soit comme un obstacle au développement, plutôt que d'en faire partie intégrante (cf. De Haas \& Rodríguez, 2010).

\section{Critiques méthodologiques}

\section{Caricatural et manque de références académiques}

Smith fait le choix d'être caricatural et de ne pas inclure d'études existantes qui projetteraient une autre lumière sur certains de ces propos. Voici cinq exemples.

Premièrement, le lecteur comprend entre les lignes que ces interventions diasporiques positives ne valent pas les effets néfastes de la mobilité pour les pays d'accueil et de destination. L'auteur ne semble pas vouloir citer d'articles académiques démontrant le renforcement mutuel du bien-être et de l'intégration d'une part, et, d'autre part, les contributions positives des activités transnationales des migrants dans les communautés d'origines (e.a. Michielsen, 2014), notamment par rapport à la résilience aux changements environnementaux (e.a. Gioli et al., 2013 ; Schöfberger, 2018).

Deuxièmement, l'auteur avance "des faits" parfois erronés, par exemple: «(...) les migrants du Sud vont aujourd'hui partout et à bord de n'importe quelle embarcation de fortune » (p. 144). Ce faisant, il ne tient pas compte des résultats des enquêtes majeures sur les migrations entre l'Europe et l'Afrique, tel que le projet MAFE. Les chercheurs y ont trouvé que la plupart des migrants arrivaient en Europe seuls et en avion, directement depuis leur pays d'origine et que le fait de voyager avec des passeurs sur des bateaux ou des pirogues reste un fait très minoritaire (Schoumaker et al., 2013).

Troisièmement, Smith avance que: "(...) le souci d'équité internationale ne saurait se confondre avec l'ouverture des frontières (...) »; "Qui compte sans son hôte compte deux fois, cela vaut aussi pour la facture de l'immigration " (p. 183). Pourtant les migrants sont aussi des producteurs et des consommateurs, des cotisants et des contribuables. Les revues de littérature concluent au même résultat: les migrations ont un impact soit nul sur l'économie - en termes d'évolution des salaires ou du chômage, ou encore de croissance économique - soit légèrement positif (e.a. Audit de l'Assemblée nationale française, 2011 ; OCDE, 2013), et un potentiel énorme reste inexploité (e.a. Banque mondiale, OCDE \& OIT, 2015).

Quatrièmement, comme le souligne Bréville (2018), Smith n'évoque nulle part le caractère inégalitaire de la croissance, qui reste «exclusive » dans les États les plus pauvres, les effets des logiques de marché, les processus d'accumulation du capital et 
d'accaparement des terres par de gros propriétaires qui détruisent l'économie paysanne en y introduisant le salariat (cf. e.a. Massey, 1988).

31 Finalement, Smith ne nous informe que très marginalement sur les politiques européennes en matière de migration, et encore moins sur les politiques africaines à ce sujet et en matière de démographie. Pourtant, ces politiques seront déterminantes.

\section{Le lien entre migration et développement économique : simpliste?} augmenter la mobilité internationale, celui-ci ne provoque pas «l'émigration» transcontinentale, avec une idée de s'installer durablement dans un autre pays. L'approche «structuraliste » avance principalement que l'inadéquation entre l'offre et la demande d'emploi, en particulier pour les jeunes, alimente cela (e.a. Lucas, 2005 ; Clemens \& Postel, 2018). Une démographie dynamique n'engendre pas mécaniquement une forte émigration. Ils migrent principalement si la croissance de la population s'accompagne d'une croissance économique lente et d'un fort taux de chômage. (...) Quand une forte croissance démographique coïncide avec une croissance économique forte, comme dans la plupart des monarchies pétrolières du Golfe, l'émigration est faible (de Haas, 2010).

En fait, si Smith cite Harding (2012), cette hypothèse n'est pas récente : elle a été avancée depuis quarante ans par le géographe Wilbur Zelinsky (1971). Son modèle a ensuite été confirmé par de nombreuses études de cas (e.a. Stark \& Taylor, 1991 ; Martin \& Taylor, 1996; Massey, 1988, 1990, 2000a, 2000b). Cette hypothèse à la base de la transition migratoire («transition dans la mobilité ») distingue plusieurs étapes : à mesure que les pays très pauvres se développent, leurs taux de mortalité, notamment infantile, chutent ; la population rajeunit et le taux d'émigration augmente. Une fois atteint un niveau de richesse élevé, les départs d'habitants diminuent et les arrivées d'étrangers s'accroissent - sauf en cas de circonstances exceptionnelles (guerre, effondrement économique, crise politique...) qui peuvent radicalement changer la donne.

Ces étapes-là peuvent être marquées par l'Indice de Développement Humain (IDH). François Héran (2018), l'ex-directeur de l'Institut National d'Études Démographiques (l'INED) en France, s'y est attelé. Héran estime irréaliste qu'à l'échéance de 2050 l'Afrique subsaharienne ait brûlé les étapes du développement pour rejoindre la position relative actuelle du Mexique (IDH 6 sur 10), ce que Smith semble penser.

\section{Chiffres mobilisés, interprétations contestées}

Héran (2018a, 2018b) critique fermement l'absence de rigueur méthodologique dans cet essai, en pointant l'inconsistance scientifique des "prévisions" et la manière fallacieuse dont il utilise les statistiques démographiques onusiennes, notamment le rapport de la division de la Population de l'ONU sur les « migrations de remplacement " (UN Population Division, 2000). Mal compris, l'ONU voulait prouver par l'absurde, sur un scénario fictif, que l'immigration ne permettait pas de contrer le vieillissement en Europe (Héran, 2018a). En reprenant des faits d'auteurs souvent de seconde main, les erreurs sont vite commises.

Contrairement à Smith, Héran (2018a) exploite «la base bilatérale des migrations » établie par la Banque mondiale, le Fonds monétaire international (FMI) et 
l'Organisation de coopération et de développement économiques (OCDE) qui compilent toutes les données existantes sur les mouvements de population reliant deux à deux les pays de la planète. Héran calcule que les Africains et leurs descendants constitueront 3 à $4 \%$ de la population européenne vers 2050 , très loin des $25 \%$ ou « 150 millions " d'Africains dont Smith prévoit l'arrivée en Europe « d'ici à 2050 » (p. 178).

Dans la même veine, les experts du Fonds Monétaire International (Gonzalez-Garcia et al., 2016) prédisent par exemple qu'en 2050 environ 34 millions de migrants originaires d'Afrique subsaharienne seront installés dans l'ensemble des 36 pays de l'OCDE (dont seulement 26 sont situés en Europe), soit $2,4 \%$ de la population totale de l'OCDE. Les démographes de l'ONU (2017) annoncent qu'entre 2015 et 2050, le solde migratoire net de l'Europe sera de 32 millions de migrants, toutes nationalités extra-européennes confondues. Une minorité seulement des migrants subsahariens (environ $15 \%$ ) gagne l'Europe.

De plus, Smith utilise sans recul et de seconde main (via un article de L'Opinion) les enquêtes compilées par l'Institut Gallup sur les intentions de migrer, qui n'ont aucune valeur prédictive (Héran, 2018b ; Migali \& Scipioni, 2018). Selon ces enquêtes, plus d'un tiers des Subsahariens aimeraient quitter leur pays. Or il faut revenir à la question posée : «Dans l'idéal, si vous en aviez l'occasion, aimeriez-vous vous installer durablement dans un autre pays ou continuer de vivre ici?». Quand les enquêteurs demandent si cela pourrait se faire dans les douze mois et, plus encore, si des préparatifs ont été engagés, les taux s'effondrent à moins de $5 \%$.

Par ailleurs, l'Organisation Internationale pour les Migrations (OIM) souligne le potentiel de la jeunesse en Afrique, qui n'est pas nécessairement tournée vers un projet migratoire (OIM, 2018).

\section{Conclusion : surtout des objectifs politiques}

Smith a finalement rendu "officiels » des constats et des perspectives qui, jusqu'ici, étaient placés sous l'étiquette de droite, voire d'extrême-droite. Bien que ce ne soit pas explicitement le message de Smith, nous dénonçons également le choix binaire selon lequel il faudrait soit se barricader, soit ouvrir les frontières (cf. Bréville, 2018 ; Brachet \& Scheele, 2018).

41 L'auteur fait référence de manière peu critique à Maurice Barrès, Robert Kaplan, Renaud Camus ou Samuel Huntington (pp. 70, 72, 106, 220) ou encore au Camp des Saints de Jean Raspail (1973), bible de référence de la droite alternative - alt-right aux EtatsUnis - et à Soumission de Houellebecq (p. 188). Par conséquent, le lecteur ressent la tentative de le rapprocher insidieusement d'une tradition idéologique dont les adeptes prédisent depuis des décennies la fin de la "civilisation occidentale», voire du «monde blanc» (e.a. p. 197).

Finalement, cet ouvrage aux airs de géographie humaine n'est probablement pas indiqué pour comprendre correctement les dynamiques, mais plutôt intéressant pour cerner des idées de la droite sur les enjeux majeurs des prochaines décennies. Comme indiqué en quatrième de couverture, cet essai assume en effet "la nécessité d'arbitrer entre intérêts et idéaux ». En revanche, il ne peut prétendre à des positions scientifiques justifiées ; cet essai n'est pas un ouvrage scientifique crédible. 


\section{BIBLIOGRAPHIE}

BRUNEL S. (2014), L'Afrique est-elle si bien partie ?, Éditions sciences humaines, 192 p.

BRÉVILLE B. (2018), « Le mythe de la ruée vers l'Europe : Immigration, un débat biaisé », Le Monde Diplomatique, $1^{\mathrm{er}}$ Nov 2018, https://groupedhistoiresociale.com/2018/11/02/immigration-undebat-biaise-benoit-breville/, consulté le 3 novembre 2018.

BRACHET J., SCHEELE J. (2018), « Stephen Smith ravive le mythe des invasions barbares, Macron et l'Académie française applaudissent », Mediapart.fr, 2 octobre 2018, https://blogs.mediapart.fr/ les-invites-de-mediapart/blog/021018/stephen-smith-ravive-le-mythe-des-invasions-barbaresmacron-et-l-academie-francaise-ap, consulté le 5 novembre 2018.

CLEMENS M.A., POSTEL H. (2018), Can Development Assistance deter Emigration?, Center for Global Development, Washington, DC, février.

DE HAAS H. (2010), Migration transitions: A theoretical and empirical inquiry into the developmental drivers of international migration, International Migration Institute, Oxford University.

DE HAAS H., RODRÍGUEZ (2010), “Mobility and Human Development: Introduction”, Journal of Human Development and Capabilities, 11, 2, pp. 177-184.

GIOLI G., KHAN T. \& SCHEFFRAN J. (2013), Remittances and community resilience to conflict and environmental hazards in Northwestern Pakistan. Remittance flows to post-conflict states: Perspective on human security and Development, Boston, Pardee Center, pp. 117-127.

GONZALEZ-GARCIA J., HITAJ E., MLACHILA M., VISETH A. \& YENICE M. (2016), "Sub-Saharan African Migration. Patterns and Spill-overs”, International Monetary Fund, 9, 19 p.

HÉRAN F. (2018a), « L'Europe et le spectre des migrations subsahariennes », Population et Sociétés, 558, Paris, septembre 2018.

HÉRAN F. (2018b), « Comment se fabrique un oracle », La Vie des idées, 18 septembre 2018, https:// laviedesidees.fr/migrations-afrique-prejuge-stephen_smith-oracle, consulté le 5 novembre 2018.

LUCAS R. (2005), International Migration and Economic Development: Lessons from Low-Income Countries, Edward Elgar Publishing, Northampton.

MICHIELSEN J., NOTTEBOOM E. \& LODEWYCKX I. (2014), Diaspora and development cooperation. A study about the role of the Antwerp diaspora from Congo, Ghana and Morocco in development cooperation and cooperation with the city of Antwerp, CeMis, commissioned by City of Antwerp, $78 \mathrm{p}$.

MIGALI S., SCIPIONI M. (2018), A global analysis of intentions to migrate, European Commission, Joint Research Centre, $57 \mathrm{p}$.

MARTIN P.L., TAYLOR J.E. (1996), “The anatomy of a migration hump,” in TAYLOR J.E. (ed.), Development strategy, employment, and migration: Insights from models, Paris, OECD, Development Centre, pp. 43-62.

MASSEY D.S. (1988), "Economic Development and International Migration in Comparative Perspective”, Population and Development Review, 14, pp. 383-413.

MASSEY D.S. (1990), "Social Structure, Household Strategies, and the Cumulative Causation of Migration”, Population Index, 56, pp. 3-26.

MASSEY D.S. (2000a), "Book Review - The Age of Mass Migration: Causes and Economic Impact by Timothy J. Hatton and Jeffrey G. Williamson", The Journal of Modern History, 72, pp. 496-497. 
MASSEY D.S. (2000b), “To study migration today, look to a parallel era”, Chronicle of Higher Education, 46, 5.

OCDE (2013), « The fiscal impact of immigration in OECD countries », International Migration Outlook, Chapter 3, https://doi.org/10.1787/migr_outlook-2013-en, pp. 125-189.

SMITH S. (2003), Négrologie : pourquoi l'Afrique meurt, Calmann-Lévy.

STARK O., TAYLOR E.J. (1991), "Migration incentives, migration types: the role of relative deprivation", The Economic Journal, 101, pp. 1163-1178.

SCHÖFBERGER I. (2018), "Environmental change and translocal vulnerability in Senegal”, German Development Institute/ Deutsches Institut für Entwicklungspolitik (DIE), Migration, Environment and Climate Change: Policy Brief Series, 3, 4, June 2018, ISSN 2410-4930.

SCHOUMAKER B., FLAHAUX M.-L., BEAUCHEMIN C., SCHANS D., MAZZUCATO V. \& SAKHO P. (2013), Changing patterns of African migration: A comparative analysis, Paris, INED, MAFE Working Paper 18.

TRIBALAT M. (2018), « Querelle François Héran/Stephen Smith », septembre 2018, http:// www.micheletribalat.fr/440919955, consulté le 25 octobre 2018.

UNITED NATIONS POPULATION DIVISION (2000), Replacement Migration: is it a Solution to Declining Ageing Populations?, New York, http://www.un.org/esa/population/unpop.htm.

UNITED NATIONS POPULATION DIVISION (2017), World Population Prospects. Key Findings \& Advance Tables, ESA/P/WP/248, Population Division United Nations, New York, 53 p.

WORLD BANK, OECD \& ILO (2015), The Contribution of Labour Mobility to economic growth, Joint paper for the $3^{\text {rd }}$ meeting of G20 Employment Working Group, Cappadocia, Turkey, 23-25 July 2015, 34 p. ZELINSKY W. (1971), "The hypothesis of the mobility transition”, Geographical Review, 61, 2, New York.

\section{NOTES}

1. Notamment : Smith S. (2003), Négrologie : pourquoi l'Afrique meurt, Calmann-Lévy, Prix Essai 2004 France Télévision.

2. Comme, par exemple, la géographe Sylvie Brunel (2014).

3. Smith S. (2018), La Ruée vers l'Europe. La jeune Afrique en route pour le Vieux Continent, Grasset, Paris, 2018. Sauf mention contraire, les citations sont tirées de cet ouvrage.

4. Smith critique, par exemple, les chiffres produits par la Banque Africaine de Développement définissant les revenus de la classe moyenne entre 2 et 20 dollars en Afrique : « Nulle part ailleurs dans le monde, ce pouvoir d'achat ne serait considéré comme un ticket d'entrée dans une classe moyenne digne de ce nom » (p. 35). 